\title{
Effects of Cilostazol and Diltiazem Hydrochloride on Ischemia- Reperfusion Injury in a Rat Hindlimb Model
}

\author{
Bekir İnan, $\mathbf{M D},{ }^{1}$ Selma Sönmez Ergün, $\mathrm{MD},{ }^{2}$ Asiye Nurten, $\mathrm{MD}, \mathrm{PhD},{ }^{3}$ \\ Canan Küçükgergin, $\mathrm{PhD},{ }^{4}$ Aslı Zengin Türkmen, $\mathrm{PhD},{ }^{3}$ Sule Seçkin, $\mathrm{PhD},{ }^{4}$ Kerem Erkalp, MD ${ }^{5}$ \\ Sedat Ziyade, $\mathrm{MD}^{6}$ \\ Departments of ${ }^{1}$ Cardiothoracic Surgery, ${ }^{2}$ Plastic, Reconstructive and Aesthetic Surgery, and ${ }^{6}$ Thoracic Surgery, \\ Bezmialem Medical School, Bezmialem Vakif University; ${ }^{3}$ Department of Physiology, Faculty of Medicine, Yeni Yuzyil \\ University; ${ }^{4}$ Department of Biochemistry, Istanbul Medical School, Istanbul University; ${ }^{5}$ Department of Anesthesiology \\ and Reanimation, Bagcilar Research and Educational Hospital, Istanbul, Turkey
}

\section{ABSTRACT}

Objective: Free radicals and neutrophils are potent sources of ischemia-reperfusion injury (I/R) and they can be limited by the use of exogenous application of some therapeutic agents. The objective of this study was to compare the effects of cilostazol and diltiazem hydrochloride in a rat hind limb model of I/R injury.

Methods: Skeletal muscles submitted to 2 hours of ischemia by placing an aneurysm clip to femoral artery and reperfused after 1, 2 and 4 hours. Seventy-two Wistar-Albino rats were randomly divided into mainly four groups according to treatment agents: Group I (control group) was treated with saline; Group II was treated with diltiazem hydrochloride; Group III was treated with cilostazol in 30\% dimethyl sulphoxide; and Group IV was treated with 30\% dimethyl sulphoxide intraperitoneally. These four main groups also subdivided into three subgroups according to duration of the reperfusion times. Blood samples were taken and all rats were sacrificed.

Results: Cilostazol-treated groups demonstrated a significant decrease in tissue and serum malondialdehyde (MDA) levels, and tissue myeloperoxidase (MPO) activity compared with other groups. Increase in serum nitric oxide (NOx) level was significantly higher in all subgroups of cilastazol, diltiazem hydrochloride, and dimethyl sulphoxide groups versus the control group.

Conclusion: Although these results suggest the beneficial effects of cilostazol and diltiazem hydrochloride on $\mathrm{I} / \mathrm{R}$ injury, the effect of cilostazol on I/R injury seems to be more efficient than diltiazem hydrochloride.

\section{INTRODUCTION}

Reperfusion is an essential prerequisite to tissue survival after critical ischemia; however, paradoxically, readmission

Received August 19, 2016; received in revised form December 8, 2016; accepted fanuary 30, 2017.

Correspondence: Selma Sönmez Ergün, Eston ebir 3. Mahalle Ilgm Sokak CD Villa 7/2, Bahcesehir, Basaksehir, Istanbul, Turkey; + 9021269152 56; fax: + 902126217580 (e-mail: selmasonmezergun@yahoo.com). of oxygen may contribute to further tissue damage and this phenomenon has been termed as reperfusion injury. With the readmission of molecular oxygen to tissue subjected to an ischemic insult, oxygen free radicals (OFRs) are formed, proinflammatory agents are produced, and circulating neutrophils accumulate. There are several potential mechanisms for neutrophil-induced injury including OFRs generation, capillary plugging, and the release of proteolytic enzymes. I/R is associated with capillary dysfunction that leads to an increased permeability during reperfusion, thus allowing proteins to escape from the vascular bed; consequently the muscles become edematous. These effects most probably originate from oxidative stress during reperfusion that is being triggered during the ischemic period by conversion of the endothelial enzyme xanthine dehydrogenase to xanthine oxidase. At the same time, OFRs cause cellular damage by inducing lipid peroxidation, which results in functional and structural cell alterations. Under normal circumstances these OFRs are counteracted by antioxidant enzymes, such as superoxide dismutase and glutathione peroxidase. However, the antioxidant enzyme system of the body is not sufficient to handle rapid-onset conditions such as I/R injury, in which high levels of OFRs and reactive nitrogen species are produced [Kalogeris 2012; Tran 2011; Blaisdell 2002].

Numerous externally administered agents to reduce I/R injury have been investigated. One of them is a synthetic cyclic adenosine monophosphate phosphodiesterase type 3 inhibitor, cilostazol. Cilostazol inhibited not only the platelet aggregation but also the neutrophil adhesion to the endothelium, and acted synergistically with endogenous prostaglandin I 2 to enhance smooth-muscle cell vasodilatation and reduce myocardial infarct size after ischemia-reperfusion injury [Iba 2006; Hakaim 1999].

The other agent is a sarcolemmal $\mathrm{Ca}^{2+}$ channel blocker, diltiazem hydrochloride. Diltiazem hydrochloride preserves mitochondrial functional ability during ischemia, and reduces neutrophil accumulation and infarct size at reperfusion [Takeo 2004; Rousseau 1991].

Diltiazem hydrochloride and cilostazol have been reported to improve myocardial recovery after ischemia-reperfusion, but their effects on skeletal muscle ischemia-reperfusion injury remain undefined. 


\section{MATERIALS AND METHODS}

\section{Animals}

Wistar albino rats (weighing 200-240 g) were kept on standard laboratory diet and tap water. All procedures were reviewed and approved by the Ethics Committee for Animal Experimentation of Istanbul University (30.03.2009/22).

\section{Clip Features}

We used an FE-752 K clip (Aesculap Yasargil-Phynox aneurysm clip) with a blade length of $11.0 \mathrm{~mm}$ to occlude the right femoral artery.

\section{Injection and Surgical Procedure}

After the induction of general anesthesia by thiopental sodium (Ibrahim Ethem Ulagay; $50 \mathrm{mg} / \mathrm{kg}$, intraperitoneally), the right lower abdominal and inguinal regions were shaved and swabbed with antiseptic solution (betadine). One horizontal cutaneous incision was made just over the inguinal crease of the right side. The right femoral artery was isolated from the surrounding connective tissue and occluded by the clip after ligation of branches from the terminal aorta to the femoral artery for a total of 2 hours. Injections of saline, diltiazem hydrochloride, cilostazol in 30\% dimethyl sulphoxide or $30 \%$ dimethyl sulphoxide were administered intraperitoneally to animals in the respective groups just before removing the clip.

Blood samples were taken from the tail veins of the rats.

\section{Experimental Protocol}

Seventy-two Wistar-albino rats were randomly assigned to mainly 4 groups (18 animals per group) according to exogenously administered agents.

These four main groups also subdivided into three subgroups (6 animals per group) according to duration of the reperfusion times (1, 2, and 4 hours).

While all 72 animals underwent the right femoral artery occlusion procedure as described above, the left femoral artery of the rats were left intact.

The animals were injected with $2 \mathrm{~mL} / \mathrm{kg}$ saline (Group I control group), $20 \mathrm{mg} / \mathrm{kg}$ of diltiazem hydrochloride (Mustafa Nevzat) (Group II diltiazem group), $20 \mathrm{mg} / \mathrm{kg}$ of cilostazol in $2 \mathrm{~mL} / \mathrm{kg} 30 \%$ dimethyl sulphoxide (Group III cilostazol group) (Abdi İbrahim and Merck, respectively) or $2 \mathrm{~mL} / \mathrm{kg}$ 30\% dimethyl sulphoxide (Group IV DMSO group).

Blood samples were taken just before the surgical procedure (serum MDA1 and serum NOx1), just before removing the aneurysm clip (serum MDA2, serum NOx2) and at the end of the duration of the reperfusion times (serum MDA3, serum NOx3) from the tail veins of the rats to measure serum MDA and serum NOx levels.

The experimental protocol is shown in Table 1 .

All rats in each group were sacrificed after the end of the durations of reperfusion times, and tissue samples were taken to evaluate tissue levels of MDA (tMDA1 for non-ischemic hindlimbs, tMDA2 for ischemic hindlimbs) and MPO (tMPO1 for non-ischemic hindlimbs, tMPO2 for ischemic hindlimbs), and to examine the tissues histologically.

\section{Biochemical Analyses}

Blood samples were centrifuged at $800 \times \mathrm{g}$ for $15 \mathrm{~min}-$ utes at $4 \mathrm{oC}$. The serum samples were stored at $-80^{\circ} \mathrm{C}$ until analysis.

The gracilis muscles were used for evaluation of MDA levels and MPO activities. The gracilis muscle samples were rinsed with cold $0.9 \%$ sodium chloride $(\mathrm{NaCl})$, blotted dry, and immediately frozen in liquid nitrogen. They were stored at $-80^{\circ} \mathrm{C}$ until assay.

\section{Serum NOx Determination}

Since NO is an unstable molecule that quickly degrades to nitrate and nitrite, we determined the total serum levels of these NO metabolites (nitrate plus nitrite termed as NOx) by using a colorimetric assay kit (Endogen, total nitric oxide assay kit code: EMSNOTOT; Thermo Fisher Scientific, Rockford, IL, USA). Nitrate was reduced to nitrite by nitrate reductase, and nitrite levels were estimated using the Griess color reagent. The levels of nitrate and nitrite are stable in blood, and the concentration of serum NOx may be an indicator of endogeneous NO formation. The results were expressed as $\mu \mathrm{mol} / \mathrm{L}$ serum.

\section{Measurement of $M D A$}

Tissue and serum MDA levels were determined by the method of Buege and Aust [Buege 1978]. The tissue samples were homogenized with ice-cold $150 \mathrm{mM}$ potassium chloride

Table 1. Experimental Protocol

\begin{tabular}{|c|c|c|c|c|c|}
\hline Group I & $2 \mathrm{~mL}$ & - & - & - & 18 \\
\hline Group II & - & $20 \mathrm{mg} / \mathrm{kg}(2 \mathrm{~mL})$ & - & - & 18 \\
\hline Group III & - & - & $20 \mathrm{mg} / \mathrm{kg}(2 \mathrm{~mL})$ & - & 18 \\
\hline Group IV & - & - & - & $2 \mathrm{~mL}$ & 18 \\
\hline
\end{tabular}

One dose of saline, diltiazem hydrochloride, cilostazol in $30 \%$ dimethyl sulphoxide or $30 \%$ dimethyl sulphoxide were administered intraperitoneally to animals in the respective groups just before removing the aneurysm clip. 
for determination of MDA levels. MDA reacts with thiobarbituric acid to give a red compound absorbing at $532 \mathrm{~nm}$. The stock reagent $(15 \% \mathrm{w} / \mathrm{v}$ trichloroacetic acid, $0.375 \% \mathrm{w} / \mathrm{v}$ thiobarbituric acid, and $0.25 \mathrm{~mol} / \mathrm{L}$ hydrochloric acid $0.01 \%$ butylated hydroxytoluene) was mixed with the sample, mixed thoroughly, and heated for $15 \mathrm{~min}$ in a boiling water bath. After cooling, the precipitate was removed by centrifugation at $1000 \times \mathrm{g}$ for $10 \mathrm{~min}$ and the absorbance of the supernatant was determined at $532 \mathrm{~nm}$ against blank containing all the reagents. The breakdown product of 1,1,3,3-tetraethoxypropane was used as standard. MDA levels were calculated using 1.56 $\times 10^{-5} \mathrm{~mol}^{-1} \mathrm{~cm}^{-1}$ as molar extinction coefficient. The protein concentrations in the tissues were measured by the bicinchoninic acid method [Smith 1985].

\section{Tissue MPO}

MPO activity was measured spectrophotometrically, according to the o-dianisidine method [Krawisz 1984]. The tissues samples were homogenized in 10 volumes of ice-cold potassium phosphate buffer $(50 \mathrm{mM}, \mathrm{pH}$ 6.0) containing $0.5 \%$ hexadecyltrimethylammonium bromide (HTAB). The homogenate was sonicated for $30 \mathrm{~s}$. After freezing and thawing three times, the homogenate was centrifuged at $15000 \times \mathrm{g}$ for $15 \mathrm{~min}$ at $4^{\circ} \mathrm{C}$. The supernatant was reacted with $50 \mathrm{mM}$ potassium phosphate buffer ( $\mathrm{pH}$ 6.0) containing $0.167 \mathrm{mg} /$ $\mathrm{mL}$ o-dianisidine dihydrochloride and $0.0005 \% \mathrm{H}_{2} \mathrm{O}_{2}$. The change in absorption was read at $460 \mathrm{~nm}$ in a spectrophotometer (UV-1201 Series; Shimadzu Scientific Instruments, Kyoto, Japan). MPO activity (1 unit) was expressed as the amount of enzyme necessary for the degradation of $1 \mu \mathrm{mol}$ of $\mathrm{H}_{2} \mathrm{O}_{2} / \mathrm{min}$ at $25^{\circ} \mathrm{C}$.

\section{Histopathological Evaluation}

Tibialis anterior muscles from ischemic and nonischemic hindlimbs were used for histopathologic evaluations. Tissue samples were fixed in 10\% formalin and embedded in paraffin. Four-micrometer-thick sections were subjected to hematoxylin-eosin staining. All slides were subjected to light microscopic examination and evaluated by a pathologist in a blind fashion. Neutrophil infiltration was evaluated to determine the severity of inflammation that resulted from ischemiareperfusion. The total number of leukocytes per high power

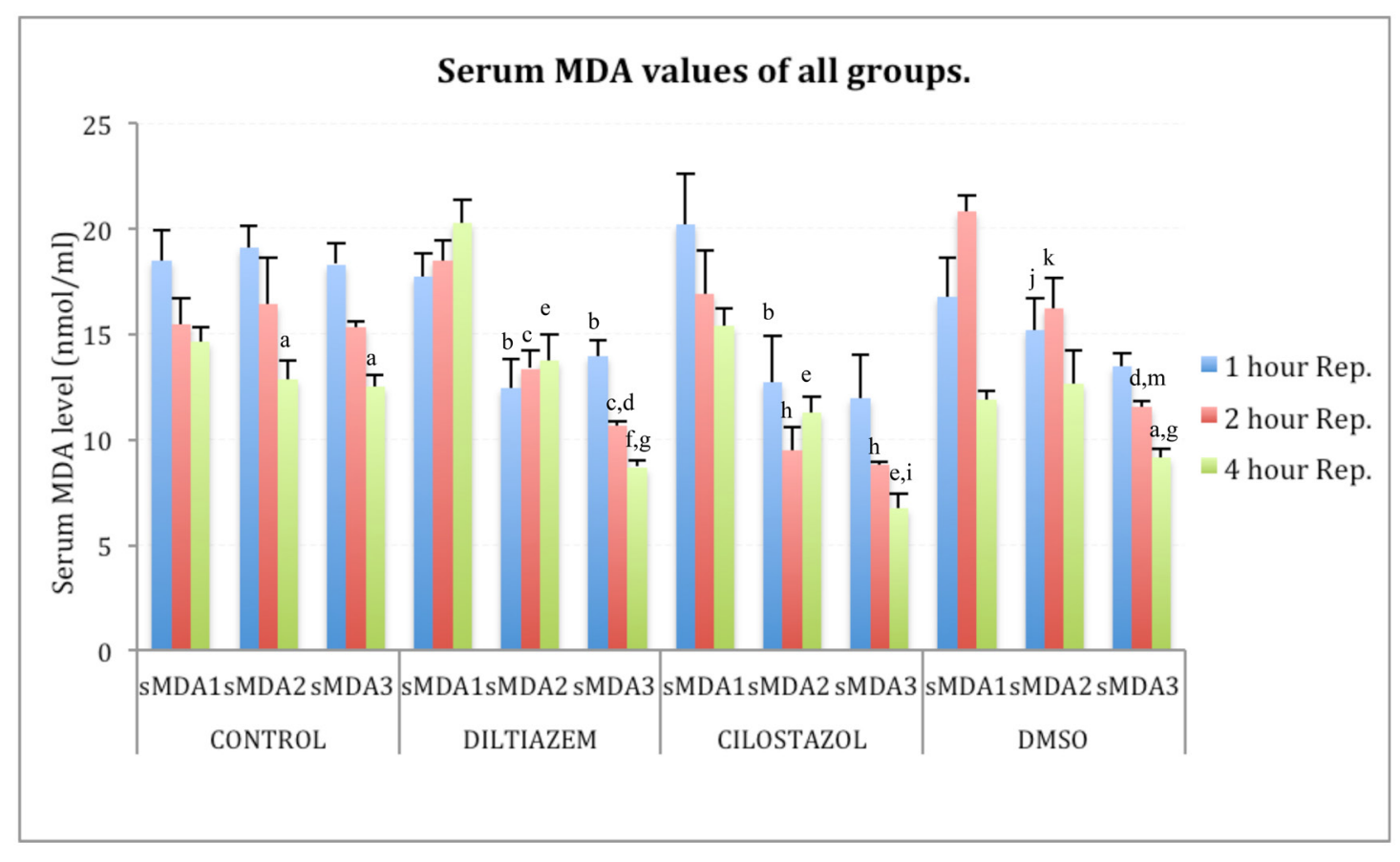

Figure 1. Serum MDA values of all groups.

sMDA1: Serum MDA value taken just before the surgical procedure; sMDA 2: Serum MDA value taken just after 2 hour ischemia period before removing the aneurysm clip; sMDA 3: Serum MDA value taken at the end of 1, 2 or 4 hour reperfusion period.

ap $<.05 ; 4$ hour rep. compared to the value of sMDA1

bP $<.05 ; 1$ hour rep. compared to the value of sMDA1

$c P<.005 ; 2$ hour rep. compared to the value of sMDA1

${ }^{d} P<.05 ; 2$ hour rep. compared to the value of sMDA2

e $P<.005 ; 4$ hour rep. compared to the value of sMDA1

${ }^{f} P<.001 ; 4$ hour rep. compared to the value of SMDA 1 $s P<.05 ; 4$ hour rep. compared to the value of sMDA2

${ }^{h} P<.02 ; 2$ hour rep. compared to the value of sMDA1

iP $<.005 ; 4$ hour rep. compared to the value of SMDA2

if $<.01 ; 1$ hour rep. compared to the value of sMDA1

$k P<.05 ; 2$ hour rep. compared to the value of sMDA1

$m p<.001 ; 2$ hour rep. compared to the value of sMDA1 


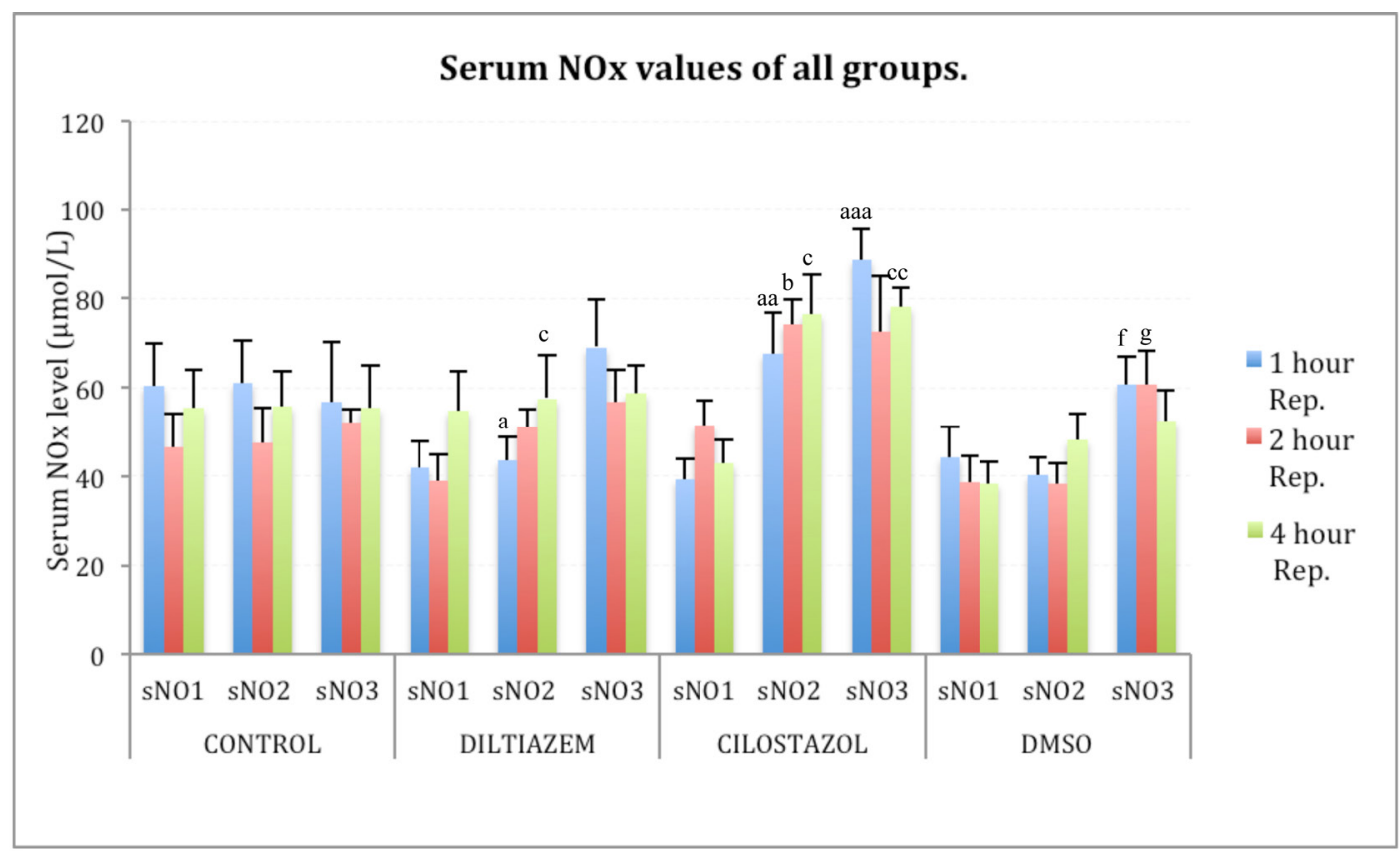

Figure 2. Serum NOx values of all groups.

sNO1: Serum NO value taken just before the surgical procedure; SNO2: Serum NO value taken just after 2 hour ischemia period before removing the aneurysm clip; sNO3: Serum NO value taken at the end of 1, 2 or 4 hour reperfusion period.

a $P<.05 ; 1$ hour rep. compared to the value of $s \mathrm{NO} 1$

aap $<.02 ; 1$ hour rep. compared to the value of $s \mathrm{NO} 1$

aap $<.005$; hour rep. compared to the value of $s \mathrm{NO} 1$

${ }^{b} P<.001 ; 2$ hour rep. compared to the value of sNO1

field (magnification 400) was counted in each section using a scale from 0 , no leukocyte infiltration; $1,<5$ leukocytes; 2 , 5-10 leukocytes; 3, >10 leukocytes. Additionally, histological changes in striation and diameters of muscle fibers, nuclear centralization, segmental necrosis, edema, and erythrocyte extravasation of skeletal muscle tissue were evaluated.

\section{Statistical Analysis}

All data were statistically evaluated by using the SPSS 15.0 program. Homogeneity differences for serum MDA and NO levels, and tissue MDA and MPO values were checked. Then, when the differences showed normal distribution, paired samples $t$ test was applied. When the differences scattered distribution, the Wilcoxon signed-rank test was applied. Pearson chi-square test was applied for histopathological data.

\section{RESULTS}

All rats remained healthy throughout the study period.

\section{Serum MDA Values}

As shown in Figure 1:

In control group. We found no significant difference between sMDA1, sMDA2, and SMDA3 values in the 1 and

\begin{abstract}
$c P<.05 ; 4$ hour rep. compared to the value of $s \mathrm{NO} 1$
cc $P<.005 ; 4$ hour rep. compared to the value of sNO1

$f P<.05 ; 1$ hour rep. compared to the value of sNO1

$s P<.05 ; 2$ hour rep. compared to the value of sNO1
\end{abstract}

2 hour reperfusion subgroups. In the 4 hour reperfusion subgroup, the $\mathrm{sMDA} 2$ and sMDA3 values were significantly decreased when compared to sMDA1 value $(P<.05)$.

In diltiazem group. There was a statistically significant decrease in $\mathrm{SMDA} 2$ and sMDA 3 values in the 1 hour $(P<.05)$ and in the 2 hour reperfusion subgroups $(P<.005)$ when compared to sMDA1 value. Also in the 2 hour reperfusion subgroup there was a statistically significant decrease in sMDA3 value when compared to $s M D A 2$ value $(P<.05)$. The sMDA2 $(P<.005)$ and sMDA3 $(P<.001)$ values were significantly decreased when compared to sMDA1 value in the 4 hour reperfusion subgroup. And the sMDA3 value was decreased when compared to the sMDA2 value in the 4 hour reperfusion subgroup $(P<.05)$.

In cilostazol group. In the 1 hour reperfusion subgroup, sMDA2 value was significantly decreased when compared to sMDA1 value $(P<.05)$. There were statistically significant decreases in sMDA2 and sMDA3 values compared to sMDA1 value in the 2 hour $(P<.02)$ and in the 4 hours reperfusion subgroups $(P<.005)$. We found significant decrease in sMDA3 value in the 4 hour reperfusion subgroup when compared to sMDA2 value $(P<.005)$.

In DMSO group. The sMDA2 value was significantly decreased when compared to the sMDA1 value in the 1 


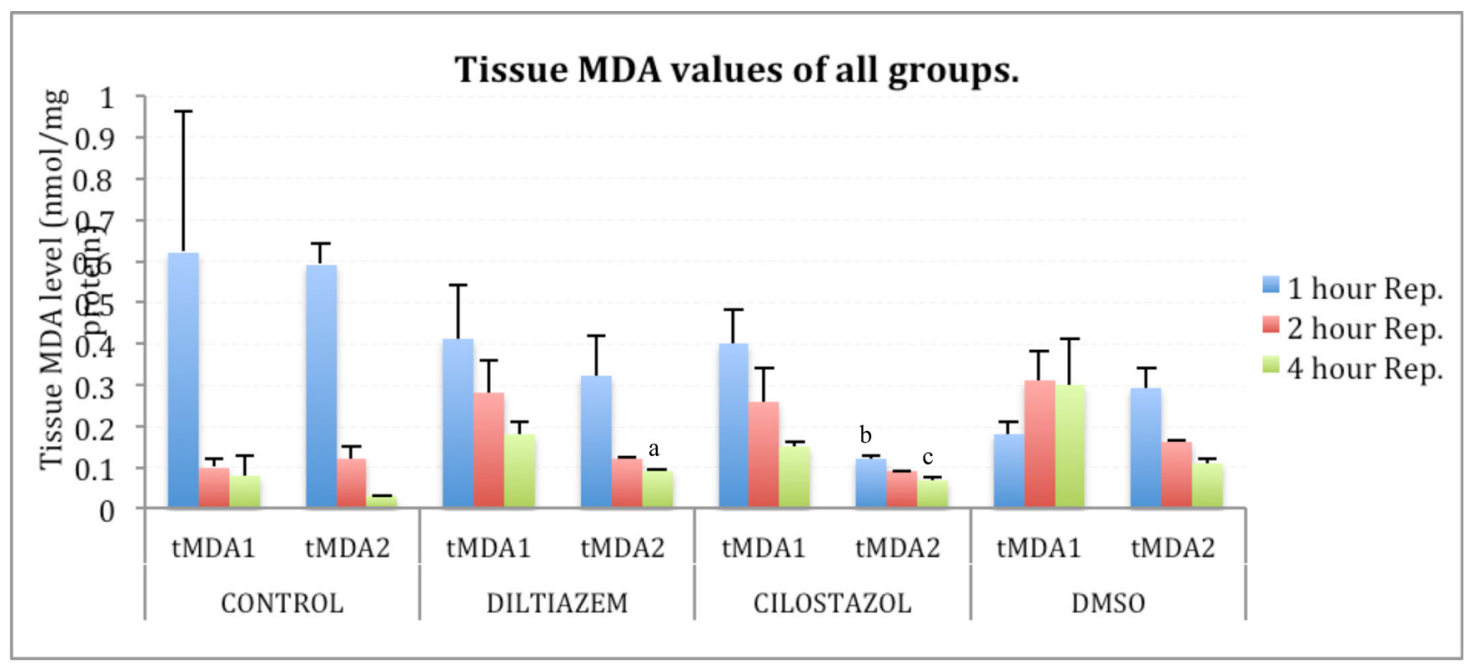

Figure 3. Tissue MDA values of all groups.

tMDA1: Tissue MDA values from non-ischemic hindlimbs.

tMDA2: Tissue MDA values from ischemic hindlimbs after 1, 2, or 4 hour reperfusion.

${ }^{\mathrm{b}} \mathrm{P}<.02 ; 1$ hour rep. according to the value tMDA1 ap $<.05 ; 4$ hour rep. according to the value tMDA1

$c P<.001 ; 4$ hour rep. according to the value tMDA1 hour reperfusion subgroup $(P<.01)$. There were statistically significant decreases in sMDA2 $(P<.05)$ and sMDA3 $(P<.001)$ values compared to sMDA1 value and in sMDA3 value $(P<.05)$ compared to sMDA2 value in the 2 hour reperfusion subgroup. In the 4 hours reperfusion subgroup, the sMDA3 value was significantly decreased when compared to sMDA1 and sMDA2 values $(P<.05)$.

\section{Serum NOx Values}

As shown in Figure 2:

In control group. There were no statistically significant differences between $\mathrm{sNO} 1$, sNOx2, and sNOx3 values in 1 , 2 , and 4 hour reperfusion subgroups.

In diltiazem group. The $\mathrm{sNO} 2$ value was significantly increased in the 1 hour reperfusion subgroup, when compared to sNOx1 value $(P<.05)$. There were no statistically significant differences between sNOx1, sNOx2, and sNOx3 values in the 2 hour reperfusion subgroup. In the 4 hours reperfusion subgroup we found a statistically significant increase in sNOx2 value compared to sNOx1 value $(P<.05)$.

In cilostazol group. There were statistically significant increases in sNOx2 $(\mathrm{P}<.02)$ and $\mathrm{sNOx} 3(P<.005)$ values compared to $\mathrm{sNO} 1$ value in the 1 hour reperfusion subgroup. The sNOx2 value was significantly increased compared to sNOx1 value $(P<.001)$ in the 2 hour reperfusion subgroup. In the 4 hours reperfusion subgroup, sNOx2 $(P<.05)$ and sNOx3 $(P<.005)$ values were significantly increased when compared to sNOx1 value.

In DMSO group. We found significant increase in sNOx3 value compared to sNOx1 value in the 1 hour reperfusion subgroup $(P<.05)$. There were statistically significant increases in sNOx3 values compared to sNOx1 values in the 2 hour reperfusion subgroup $(P<.05)$. No significant differences between sNOx1, sNOx2 and sNOx3 values were found in the 4 hours reperfusion subgroup.

\section{Tissue MDA Values}

As shown in Figure 3:

In control group. There was no significant difference between $\mathrm{tMDA} 1$ and $\mathrm{tMDA} 2$ values in 1, 2, and 4 hour reperfusion subgroups.

In diltiazem group. There was no significant difference between tMDA1 and tMDA2 values in the 1 and 2 hour reperfusion subgroups. In the 4 hour reperfusion subgroup, tMDA2 value was significantly decreased when compared to tMDA1 value $(P<.05)$.

In cilostazol group. There was a statistically significant decrease in the tMDA2 value compared to sMDA1 values in the 1 hour $(P<.02)$ and in the 4 hour reperfusion subgroups $(P<.001)$.

In DMSO group. We found no difference between tMDA1 and tMDA2 values in 1, 2, and 4 hour reperfusion subgroups.

\section{Tissue MPO Activities}

As shown in Figure 4:

In control group. There were no significant differences between tMPO1 and tMPO2 values in the 1, 2 and 4 hour reperfusion subgroups.

In diltiazem group. We found no difference between tMPO1 and $\mathrm{tMPO} 2$ values in the 1, 2, and 4 hour reperfusion subgroups. 


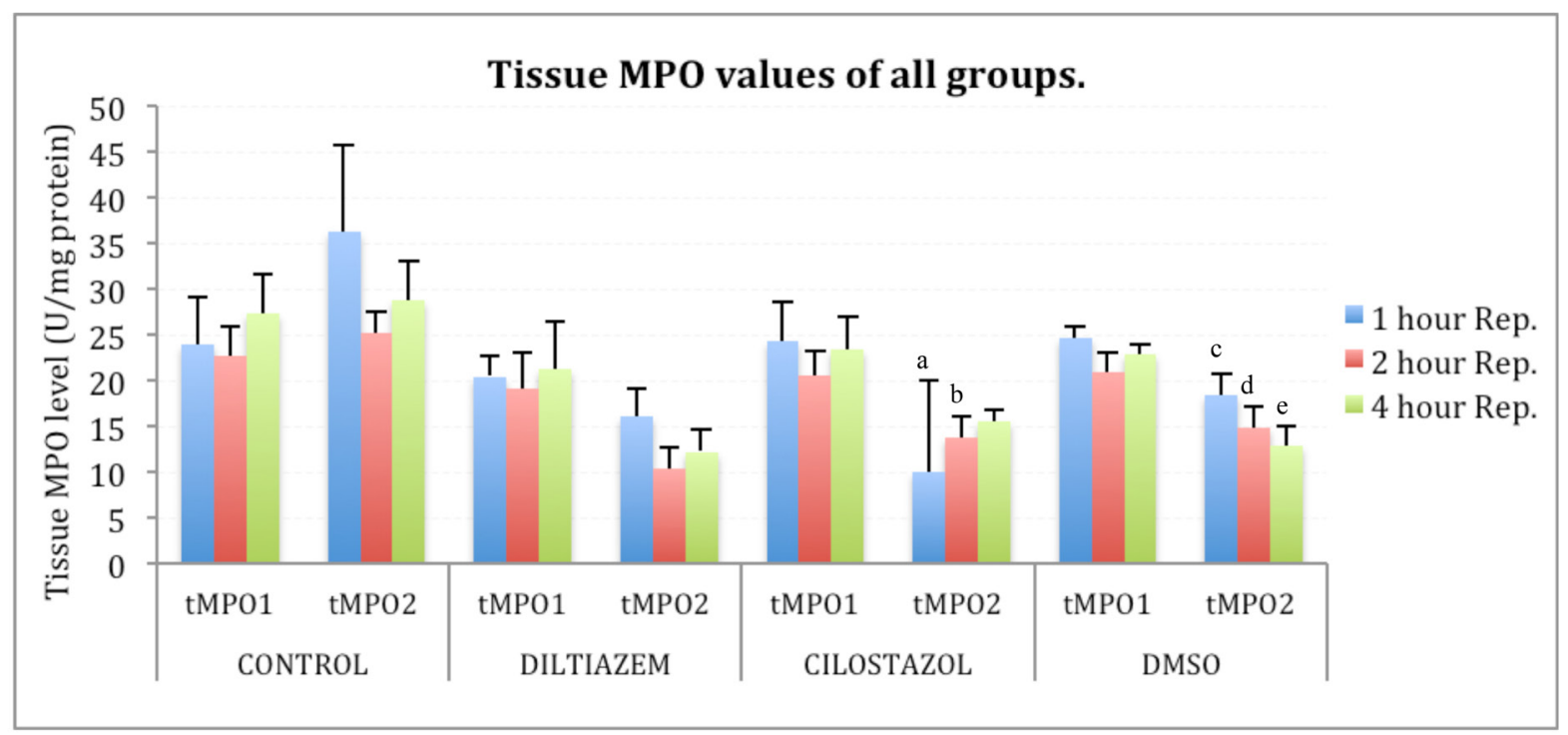

Figure 4. Tissue MPO activities of all groups.

tMPO1: Tissue MPO activities from non-ischemic hindlimbs.

tMPO2: Tissue MPO activities from ischemic hindlimbs after 1, 2 or 4 hour reperfusion.

aP $<.02 ; 1$ hour rep. according to tMPO1 activity

${ }^{\mathrm{b} P}<.05 ; 2$ hour rep. according to tMPO1 activity

$c P<.051$ hour rep. according to tMPO1 activity

${ }^{\mathrm{d}} P<.02 ; 2$ hour rep. according to tMPO1 activity

eP $<.005 ; 4$ hour rep. according to tMPO1 activity

In cilostazol group. The tMPO2 value was significantly decreased in the 1 hour $(P<.02)$ and in the 2 hour reperfusion subgroups $(P<.05)$ when compared to tMPO1 value. There was no difference in the 4 hour reperfusion subgroup.

In DMSO group. There was a statistically significant decrease in tMPO2 value in the 1 hour $(P<.05), 2$ hour $(P<.02)$, and in the 4 hour reperfusion subgroups $(P<.005)$ when compared to tMPO1 value.

\section{Histopathology}

As shown in Table 2:

Neutrophil infiltrations in all ischemic groups, in all nonischemic groups, and in both groups were compared with each other. Therewere nostatistically significant differences between the ischemic groups. In all non-ischemic groups, neutrophil infiltrations were indifferent between all non-ischemic groups. Neutrophil infiltrations of all ischemic and non-ischemic groups were not statistically significant between groups.

\section{DISCUSSION}

Long periods of ischemia dramatically decrease the capacity of regenerating adenosine triphosphate (ATP) through the aerobic systems. As a result, skeletal muscle cells must alter from oxidative phosphorylation to anaerobic glycolysis for energy production. The glycolytic regeneration of ATP necessitates the decomposition of adenosine monophosphate (AMP) that further degrades to hypoxanthine and uric acid.
Increased break down of adenine nucleotides and inhibition of xanthine oxidase activity in deficiency of oxygen leads to accumulation of hypoxanthine in tissues. Accumulated hypoxanthine may serve as substrate for increased superoxide radical production by xanthine oxidase. OFRs cause cellular damage and further tissue necrosis by inducing lipid peroxidation, which results in functional and structural cell alterations. Formation of OFRs, production of proinflammatory agents, and accumulation of circulating neutrophils eventuate with the readmission of molecular oxygen to tissue subjected to an ischemic insult [Kalogeris 2012; Blaisdell 2002; Granger 2015; Östman 2004].

In the study, ischemia was induced via microvascular clamping of the pedicle to mimic the situations encountered in clinical practice such as complex wound reconstructions and limb replantation procedures. Due to the maximally allowed normothermic ischemia time of two hours in limb surgery, our particular interest was focused on the two hour ischemia [Turchányi 2005].

Formerly, it was considered that cellular injury associated with $\mathrm{I} / \mathrm{R}$ injury is primarily a result of the ischemic insult; nowadays it is apparent that $\mathrm{I} / \mathrm{R}$ injury mainly occurs during the reperfusion phase due to the production of OFRs and accumulation of neutrophils. For that reason, therapeutic strategies are directed toward either limiting the production of OFRs or detoxifiying these metabolites after they are produced, and with neutrophil depletion or prevention of neutrophil adherence by therapeutic agents [Ferrari 2015]. 
Table 2. Histopathologic Values of All Groups

\begin{tabular}{lccc}
\hline Control & $\begin{array}{c}1 \text { hour rep. } \\
(\text { mean } \pm \text { SD) }\end{array}$ & $\begin{array}{c}2 \text { hour rep. } \\
(\text { mean } \pm \text { SD) }\end{array}$ & $\begin{array}{c}\text { 4 hour rep. } \\
\text { (mean } \pm \text { SD })\end{array}$ \\
\hline $\begin{array}{l}\text { Non-ischemic }(n=6) \\
\text { Ischemic }(n=6)\end{array}$ & $0.33 \pm 0.5$ & $0.33 \pm 0.5$ & $0.33 \pm 0.5$ \\
Diltiazem & $1.50 \pm 0.5$ & $1.67 \pm 0.5$ & $1.83 \pm 0.4$ \\
$\quad$ Non-ischemic $(n=6)$ & $0.33 \pm 0.5$ & $0.33 \pm 0.5$ & $0.33 \pm 0.5$ \\
Ischemic $(n=6)$ & $1.33 \pm 0.5$ & $1.33 \pm 0.5$ & $1.33 \pm 0.5$ \\
Cilostazol & & & \\
Non-ischemic $(n=6)$ & $0.33 \pm 0.5$ & $0.33 \pm 0.5$ & $0.33 \pm 0.5$ \\
Ischemic $(n=6)$ & $1.33 \pm 0.5$ & $1.17 \pm 0.4$ & $1.17 \pm 0.4$ \\
DMSO & & & \\
$\quad$ Non-ischemic $(n=6)$ & $0.33 \pm 0.5$ & $0.33 \pm 0.5$ & $0.33 \pm 0.5$ \\
Ischemic $(n=6)$ & $1.33 \pm 0.5$ & $1.50 \pm 0.5$ & $1.50 \pm 0.5$ \\
\hline
\end{tabular}

There were no statistically significant differences between ischemic and non-ischemic groups.

It has also been noted that the histological appearance of reperfusion injury to skeletal muscle is patchy distribution due a large number of fibers being unaffected. For that reason, control of inflammation by using therapeutic agents may lead to meaningful muscle recovery due to patchy nature of injury [Chan 2004].

Cilostazol inhibits platelet aggregation by increasing platelet cAMP concentration, increasing NO production through a cAMP-dependent pathway, increasing smooth-muscle cell vasodilatation by acting synergistically with endogenous prostaglandin $\mathrm{I}_{2}$, promoting neo-vascularization in response to tissue ischemia via an eNOS-dependent mechanism, and reducing myocardial infarct size after I/R injury [Iba 2006; Hakaim 1999; Hori 2012].

Cilostazol also has a clinically beneficial effect in patients with intermittent claudication by increasing microvascular circulation. Intermittent claudication is the most common form of chronic ischemia of the extremities and hypothetically intermittent claudication may represent a subclinical I/R [Santos 2012; Miyashita 2011].

Diltiazem hydrochloride preserves mitochondrial functional ability, inhibits the increase of non-esterified fatty acids and intracellular calcium overload during ischemia, and reduces neutrophil accumulation and infarct size at reperfusion [Takeo 2004; Rousseau 1991].

Based on the findings mentioned above we investigated the effect of cilostazol and diltiazem hydrochloride on I/R injury in the rats due to platelet aggregation, neutrophil adhesion, thrombosis and vasoconstriction as components of I/R injury.

Muscle fibers are classified as type I and type II fibers based on the amount of myoglobin content of the muscle. While type I fibers have predominantly oxidative metabolism of triglycerides as their energy source, type II fibers have anerobic metabolism of glycogen as their main energy source. Type
I fibers are more vulnerable to ischemia than type II fibers [Östman 2004; Lindsay 1990]. We chose tibialis anterior muscles for histopathological examinations due to the tibialis anterior muscle containing predominantly type I fibers, which reflects the effects of ischemia better than other fiber types.

MDA, the product of peroxidation of membrane polyunsaturated and saturated fatty acids, indicates oxidative damage [Buege 1978]. For that reason, serum and tissue MDA concentrations were measured to evaluate the impact of I/R injury on skeletal muscle lipid peroxidation. Cilostazol treatedgroup III demonstrated a significant decrease in serum MDA levels at 2 and 4 hours of reperfusion time and in tissue MDA levels at 1 and 4 hours of reperfusion time. Diltiazem treatedgroup II demonstrated a significant decrease in serum MDA levels at all reperfusion times and in tissue MDA levels at only 4 hour reperfusion time.

Serum NO concentrations were measured due to OFRs by directly inactivating the endogenous vasodilator, leading NO to vasoconstriction by impairing local $\mathrm{NO}$ mechanisms that control the vessel tone [Parvuvums 1999]. Increase in sNOx activity was significantly higher in cilostazol-treated group III versus the other groups.

tMPO activity was determined as an index of PMN accumulation [Kadambi 2000]. There were significant decreases in tissue myeloperoxidase activities in cilastazol treated-group III.

No significant difference between all subgroups of ischemic and non-ischemic groups was noted in histopathological findings. It has been difficult to determine when muscle dies due to gross and microscopic changes being minimal for many hours following muscle death. Traditional histology does not provide definitive evidence of muscle destruction if the ischemia/reperfusion time is not long enough, because longer ischemia leads to more serious tissue damage, and inflammatory mediators and inflammatory reaction act later. It may be possible to demonstrate the muscle damage with more advanced techniques such as new spectrophotometric techniques that permit such documentation. It was reported that significant muscle injury occurring after three hours of ischemia can be demonstrated using spectrophotometric triphenyltetrazolium chloride analysis of skeletal muscle [Östman 2004; Rácz 1996; Schlag 2001]. Therefore, with tissue samples taken just after the reperfusion time for histopathological evaluation and neutrophil infiltrations used to indicate muscle injury, we may have found these results.

In conclusion, the present study demonstrated that the effect of cilostazol on I/R injury seems to be more efficient than diltiazem hydrochloride. The action of cilostazol should be clarified by further investigation since cilostazol could become a leading compound for investigation of protection against skeletal muscle I/R injury.

\section{REFERENCES}

Blaisdell FW. 2002. The pathophysiology of skeletal muscle ischemia and reperfusion syndrome: a review. Cardiovasc Surg 10:620-30.

Buege JA, Aust SD. 1978. Microsomal lipid peroxidation. In: Methods in enzymology. P Fleischer, L Packer (eds). New York: Academic Press. vol. 52 , pp. 302-10. 
Chan RK, Austen WG Jr, Ibrahim S, et al. 2004. Reperfusion injury to skeletal muscle affects primarily type II muscle fibers. J Surg Research 122:54-60.

Ferrari RS, Andrade CF. 2015. Oxidative stress and lung ischemia-reperfusion injury.

Oxid Med Cell Longev 2015:590987.

Granger DN, Kvietys PR. 2015. Reperfusion injury and reactive oxygen species: The evolution of a concept. Redox Biol 6:524-51.

Hakaim AG, Cunningham L, White JL, Hoover K. 1999. Selective type III phosphodiesterase inhibition prevents elevated compartment pressure after ischemia/reperfusion injury. J Trauma 46:869-72.

Hori A, Shibata R, Morisaki K, Murohara T, Komori K. 2012. Cilostazol stimulates revascularisation in response to ischemia via an eNOS-dependent mechanism. Eur J Vasc Endovasc Surg 43:62-5.

Iba T, Kidokoro A, Fukunaga M, Takuhiro K, Ouchi M, Ito Y. 2006. Comparison of the protective effects of type III phosphodiesterase (PDE3) inhibitor (cilostazol) and acetylsalicylic acid on intestinal microcirculation after ischemia reperfusion injury in mice. Shock 26:522-6.

Kadambi A, Skalak TC. 2000. Role of leukocytes and tissue-derived oxidants in short-term skeletal muscle ischemia-reperfusion injury. Am J Physiol Heart Circ Physiol 278:H435-43.

Kalogeris T, Baines CP, Krenz M, Korthuis RJ. 2012. Cell biology of ischemia/reperfusion injury. Int Rev Cell Mol Biol 298:229-317.

Krawisz JE, Sharon P, Stenson WF. 1984. Quantitative assay for acute intestinal inflammation based on myeloperoxidase activity. Assessment of inflammation in rat and hamster models. Gastroenterology 87:1344-50.

Lindsay TF, Liauw S, Romaschin AD, Walker PM. 1990. The effect of ischemia/reperfusion on adenine nucleotide metabolism and xanthine oxidase production in skeletal muscle. J Vasc Surg 12:8-15.

Miyashita Y, Saito S, Miyamoto A, Iida O, Nanto S. 2011. Cilostazol increases skin perfusion pressure in severely ischemic limbs. Angiology 62:15-7.

Östman B, Michaelsson K, Rahme H, Hillered L. 2004. Tourniquet-induced ischemia and reperfusion in human skeletal muscle. Clin Orthop 418:260-5.

Parvuvums DV. 1999. The pathology of ischemia-reperfusion. In: Ischemia-reperfusion injury. PA Grace, RT Mathie (eds). London: Blackwell Science. pp. 3-19.

Rácz IB, Sarkadi L, Hamar J. 1996. The functional damages of ischemia/ reperfused skeletal muscle. Acta Physiol Hung 84:205-16.

Rousseau G, St-Jean G, Latour JG, Merhi Y, Nattel S, Waters D. 1991. Diltiazem at reperfusion reduces neutrophil accumulation and infarct size in dogs with ischaemic myocardium. Cardiovasc Res 25:319-29.

Santos MR, Celotto AC, Capellini VK, Evora PR, Piccinato CE, Joviliano EE. 2012. The protective effect of cilostazol on isolated rabbit femoral arteries under conditions of ischemia and reperfusion: the role of the nitric oxide pathway. Clinics (Sao Paulo) 67:171-8.

Schlag MG, Harris KA, Potter RF. 2001. Role of leukocyte accumulation and oxygen radicals in ischemia-reperfusion-induced injury in skeletal muscle. Am J Physiol Heart Circ Physiol 280:H1716-21.

Smith PK, Krohn RI, Hermanson GT, et al. 1985. Measurement of protein using bicinchoninic acid. Anal Biochem 150:76-85.

Takeo S, Tanonaka K, Iwai T, Motegi K, Hirota Y. 2004. Preservation of mitochondrial function during ischemia as a possible mechanism for cardioprotection of diltiazem against ischemia/reperfusion injury. Biochem Pharmacol 67:565-74.

Tran TP, Tu H, Pipinos II, Muelleman RL, Albadawi H, Li YL. 2011. Tourniquet-induced acute ischemia-reperfusion injury in mouse skeletal muscles: involvement of superoxide. Eur J Pharmacol 650:328-34.

Turchányi B, Tóth B, Rácz I, Vendégh Z, Furész J, Hamar J. 2005. Ischemia reperfusion injury of the skeletal muscle after selective deafferentation. Physiol Res 54:25-31. 